\section{Association between Early Hyperoxia and Neurologic Outcome in Patient with High Grade Aneurysmal Subarachnoid Hemorrhage}

\section{Abstract}

Background: Oxygenation support is a common practice in patients with aneurysmal subarachnoid hemorrhage (SAH). The aim of this study was to analyze the association between early hyperoxia and the neurological outcome in patients presenting a High Grade SAH, using the modified Rankin Scale (mRS) at 6 months.

Methods: We retrospectively analyzed data of SAH in a single center, retrospective, observational study. The inclusion criteria were patients' $\geq 18$ years-old, under mechanical ventilation upon admission to the intensive care unit (ICU) and presenting an aneurysmal SAH with a WFNS score $\geq 3$. Hyperoxia was defined as $\mathrm{PaO}_{2}>120 \mathrm{mmHg}$ and classified into 3 subgroups: mild $(121-200 \mathrm{mmHg}$ ), moderate (201-300 $\mathrm{mmHg}$ ) and severe (> $300 \mathrm{mmHg})$. Patients with a 6 months$\mathrm{mRS}>3$ were considered as having a poor outcome.

Results: One hundred and thirty-nine patients with aneurysmal SAH were included. $82 \%$ of patients were exposed to hyperoxia with predominance of mild hyperoxia (42.8\%). A higher yet non-statistical, poor neurologic outcome was identified in the hyperoxic compared to normoxic group (72\% vs. 87.7\% OR 2.8 [1.02-7.71], p 0.12 ). Following a multivariate analysis, a statistical trend was identified between the hyperoxic group and a 6-month poor neurological outcome (OR 2.84, 95\% CI, $0.99-8.19 ; p=0.052)$, but no statistical association was found with delayed cerebral ischemia (OR 1.68, 95\% Cl, 0.5-5.71, $\mathrm{p}=0.39$ ) nor with 28-day mortality (OR 2.17, $95 \% \mathrm{Cl}, 0.82-5.75, \mathrm{p}=0.11)$.

Conclusions: Our findings suggest a statistical trend between early hyperoxia and a poor neurological outcome at 6 months, in patients with a high grade aneurysmal $\mathrm{SAH}$ admitted to the ICU. Further, large-scale studies are required to fully evaluate the effects of hyperoxia.

Keywords: Aneurysmal sub-arachnoid hemorrhage; Hyperoxia; Outcomes; Intensive care

\section{Camille Beaudeux', Lukshe Kanagaratnam², Mathieu Bard', Habib Habchi ${ }^{3}$, Benoit Marlier, Jean-Charles Kleiber ${ }^{4}$ and Vincent Legros ${ }^{\text {* }}$}

1 Department of Anesthesiology and Critical Care, White House University Hospital of Reims, Reims Cedex, France

2 Department of Clinical Research, Robert Debré University Hospital of Reims, Reims Cedex, France

3 Department of Emergency, White House University Hospital of Reims, Reims Cedex, France

4 Department of Neurosurgery, White House University Hospital of Reims, Reims Cedex, France

5 Surgical and Trauma Intensive Care Unit, White House University Hospital of Reims, Reims Cedex, France

*Corresponding author: Dr. Vincent Legros

vlegros@chu-reims.fr

Surgical and Trauma Intensive Care Unit, White House University Hospital of Reims, 45, Rue Cognacq Jay, 51092 Reims Cedex, France.

Tel: +33-03-26-78-30-21

Fax: +33-03-26-78-48-49

Citation: Beaudeux C, Kanagaratnam L, Bard M, Habchi H, Marlier B, et al. (2019) Association between Early Hyperoxia and Neurologic Outcome in Patient with High Grade Aneurysmal Subarachnoid Hemorrhage. J Neurol Neurosci Vol.10 No.3:299

Initial management requires airway control to prevent hypoxia. Frequently, this oxygen supplementation leads to hyperoxia. This hyperoxia is responsible for a production of free radicals that would be harmful. To our knowledge, few studies investigated the impact of hyperoxia on the neurological outcome of patients 
with SAH [7-9], and its underlying mechanisms remain unclear. One study showed an increased rate of cerebral delayed ischemia $(\mathrm{DCl})$, as well as a worsening neurological prognosis in patients subjected to hyperoxia [8]. We, therefore, hypothesized that hyperoxia at ICU admission, exacerbated the neurological outcome of patients with high grade $\mathrm{SAH}$ requiring neurointensive care and mechanical ventilation. Consequently, we sought to analyze the relation between hyperoxia upon ICU admission, and the neurological outcome in high grade SAH patients, using the modified Rankin Scale (mRS) at 6 months after stroke.

\section{Patients and Methods}

This single-center, retrospective cohort study was conducted at Reims University Hospital, a regional reference center, with an annual ICU admission rate of 30 to 50 patients with SAH. Hospitalized SAH patients received interdisciplinary medical care, with a team of specialists including a neurosurgeon, a neuroradiologist, an anesthesiologist and a neuro-intensivist within the ICU.

This study was undertaken in accordance with the Declaration of Helsinki and the French legislation on ethics in biomedical research. It was approved by the institutional review board (IRB) of the French Society of Anesthesiology and Critical Care (SFAR) (Approval number: 00010254---2018---090), as well as the Commission Nationale de I'Informatique et des Libertés (CNIL) (authorisation number: 2004721 v 0).

\section{Inclusion criteria and population}

Patients included were, 18 years of age and older, admitted in the ICU between January 2012 and April 2018, with a confirmed diagnosis of an aneurysmal SAH with a WFNS score $\geq 3$, and requiring mechanical ventilation. Pregnant women and patients presenting a traumatic SAH were excluded.

\section{Management of SAH patients in ICU}

All patients were managed in accordance with the Recommendations from the Neurocritical Care Society's Multidisciplinary Consensus Conference [7]. Patients were monitored on a case-by-case basis with, an Intracranial Pressure probe, as well as an oxygenation monitoring by a $\mathrm{PtBO}_{2}$ probe. The cerebral vascularization was also monitored at least 3 times a day using a transcranial Doppler (TCD).

Delayed Cerebral Ischemia (DCI) was defined using admitted criteria and TCD findings of $\mathrm{DCl}$ for patient under mechanical ventilation, and for association between TCD signs and development of new focal neurological sign and confirmed by appearance of new infarcts on computed tomography, resonance imaging or cerebral angiography [8]. Fluid management was administered according to recommendations, analgesia was achieved using sufentanil or remifentanil, and sedation was obtained using Midazolam or Propofol, and rapidly interrupted once the SAH was secured by procedure (surgery or coiling) and with no consequent episodes of intracranial hypertension (ICH) or ventilator dysynchrony [7].

The treatment of $\mathrm{ICH}$ followed the international guidelines [7]. Patients with refractory $\mathrm{ICH}$ may have been treated with therapeutic hypothermia $\left(35^{\circ} \mathrm{C}\right)$, after careful review and in conjunction within the medical team. Patients received Nimodipine as well via the nasogastric tube in prevention of $\mathrm{DCl}$, and hyperthermia was treated by acetaminophen or external cooling.

Mechanical ventilation was delivered in a volume-controlled mode with constant inspiratory flow, with tidal volume targeted at $6 \mathrm{ml}$ per kilogram of predicted body weight, and the PEEP level between 5 and $12 \mathrm{~cm} \mathrm{H}_{2} \mathrm{O}$ and $\mathrm{FiO}_{2}$ for objective $\mathrm{PaO}_{2}$ between 80 and $120 \mathrm{mmHg}$, respiratory rate for $\mathrm{PCO}_{2}$ range to $38-42 \mathrm{mmHg}$.

\section{Data recording}

The following data were collected: Age, sex, chronic hypertension, tobacco and/or alcohol use, body temperature, $\mathrm{PaO}_{2}$ at admission coma Glasgow scale (CGS), Fisher scale and WFNS grading, presence of a securing procedure, the simplified acute physiology score (SAPS II), episodes of ICH (Intra cranial hemorrhage), seizures, hydrocephaly, documented ventilator-acquired pneumonia, cardiac dysfunction, events of $\mathrm{DCl}$, of pulmonary neurogenic edema, as well as sepsis, duration of mechanical ventilation and ICU length of stay, mortality at $28^{\text {th }}$ day post admission, 6-month mRS score.

\section{Definitions of hyperoxia}

We collected $\mathrm{PaO}_{2}$ at the admission of patients under mechanical ventilation. Hyperoxia was defined as a $\mathrm{PaO}_{2}$ value above 120 $\mathrm{mmHg}$ like a previous study [8]. We then defined 3 subgroups of hyperoxia i.e., mild (121-200 $\mathrm{mmHg})$, moderate $(201-300 \mathrm{mmHg}$ ) and severe $(>300 \mathrm{mmHg}$ ) as previously described, to examine the effect of hyperoxia exposure in mechanically ventilated patient) [10].

\section{Complications}

Ventilator-associated pneumonia was identified following the French recommendations [11] and $\mathrm{ICH}$ was defined as an Intracranial Pressure (ICP) value above $25 \mathrm{mmHg}$, for more than 5 minutes. Cardiac dysfunction was assessed by a left ventricular ejection fraction (LVEF) below $50 \%$, and pulmonary neurogenic edema was considered, in the absence of increased left ventricular pressure, along with the presence of B-lines in pulmonary ultrasonography or edema on chest X-ray, and the absence of any other probable cause for developing an Acute Respiratory Distress Syndrome (ARDS) [12]. Sepsis was also evaluated according to the criteria of the Surviving Sepsis Campaign [13].

\section{Outcome}

The main end point was the mRS score at 6 months (+/- 2 weeks), which was either evaluated during a follow-up consultation or by telephone interview. A poor neurological prognosis was defined by a $\mathrm{mRS}$ value $>3$ at 6 months. Secondary outcomes included $28^{\text {th }}$ day mortality, the presence of $\mathrm{DCl}$, the duration of mechanical ventilation, and ICU-hospitalization period.

\section{Statistical analysis}

We performed a descriptive analysis of the data collected in our study. Qualitative variables are described as numbers (percentages), whereas quantitative variables as mean \pm standard deviation or median and interquartile ranges. Associations 
between $\mathrm{PaO}_{2}$ levels and demographic and clinical baseline data were investigated using the Chi-squared test, Fisher's exact test, or Kruskal Wallis test as deemed appropriate. The association between hyperoxia and the outcomes "poor prognosis", DCl, and $28^{\text {th }}$ day mortality was examined using a bivariate analysis, and odds ratios (OR) with 95\% Confidence Interval (95\% Cl) were calculated. Multivariable logistic regression analysis was performed, correcting adjustments for age, sex, poor prognosis, $\mathrm{DCl}$ events and their treatment, and for mortality day-28. Results bearing $p$-value $<0.05$ were considered as statistically significant. All analyses were performed using SAS, version 9.4 (SAS Institute, Inc., Cary, NC, USA).

\section{Results}

\section{General characteristics of the population}

Between January 2012 and April 2018, 195 patients were admitted into the ICU under mechanical ventilation with a confirmed diagnosis of SAH. 56 of the 195 patients were excluded (1 for age $<18$ years, 29 for a WFNS grading $<3$, and 25 for traumatic $\mathrm{SAH}$ ) (Figure 1). A total of 139 patients with aneurysmal high grade SAH were therefore analyzed. Ninety-five patients (68\%) patients were women with a mean age of 54.9 years old. Chronic hypertension and tobacco use were present in at least one third of patients. All characteristics are summarized in Table 1. A WFNS grade of 5 and a Fisher scale of 4 were mainly identified in our cohort. One third of all aneurysm treatment was performed by interventional neuroradiology. Seventy-three percent of patient had at least one episode of high ICP measure, and 23\% developed a DCI during their ICU hospitalization period. The SAPS II median was 56 (45-64), while the median mechanical ventilation duration was 11 days. $28^{\text {th }}$ day mortality was about $49.6 \%$ and surged to $66 \%$ at 6 months.

\section{Hyperoxia subgroups}

The mean $\mathrm{PaO}_{2}$ at admission was $181 \mathrm{mmHg}$ (Table 1). In our study $82 \%$ of patients were exposed to hyperoxia at admission, with the mild hyperoxia subgroup $\left(\mathrm{PaO}_{2}[121-200 \mathrm{mmHg})\right.$ being

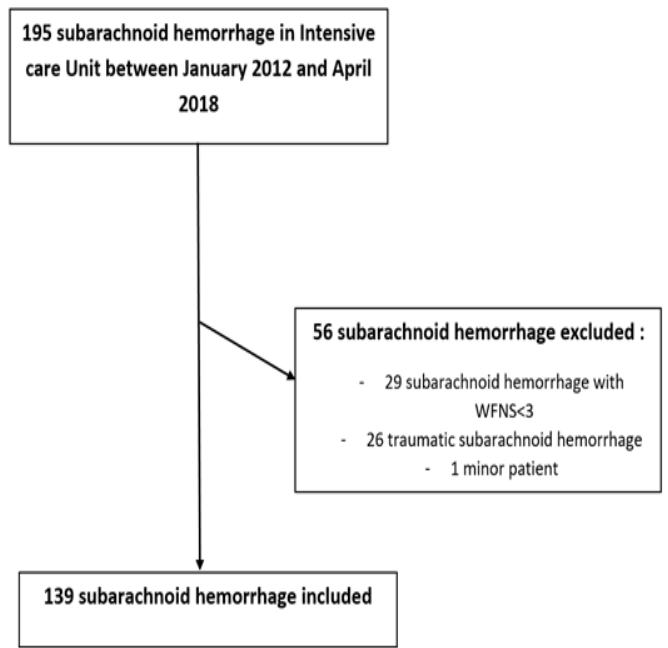

Figure 1 Flow chart. the mostly represented (42.8\%) (Table 2). Univariate analysis reported no significant differences in age, sex, medical history, treatment and adverse events. The ICU mortality and mRS scores were not statistically different among the 3 subgroups, and the 6-months mortality ( $m R S=6$ ) ranged between 48 and $71.4 \%$. However, a significant correlation was noted between higher WFNS scores and moderate to severe hyperoxia $(p=0.0005)$ (Table 2). There were also more episodes of High-ICP in hyperoxia subgroups.

\section{Neurological outcomes}

Poor neurologic prognosis was more predominant in the hyperoxia vs. normoxia $(87.7 \%$ vs. $72 \%$ OR 2.8 [1.02-7.71]; $\mathrm{p}=0.12$ ), though not statistically related. In multivariate analysis however, a statistical trend was identified between hyperoxia and the emergence of a poor neurologic outcome (OR=2.84, [0.998.19]; $p=0.052$ ) (Table 3).

\section{Secondary outcomes}

$\mathrm{DCl}$ episodes, as well as $28^{\text {th }}$ day mortality, were both more predominant in the hyperoxia vs. normoxia group, with the latter outcome being statistically different (32\% vs. 54\%, OR 2.5 [0.93-7.31]; $p=0.05)$. In multivariate analysis, hyperoxia was not significantly associated with $\mathrm{DCl}(\mathrm{OR}=1.68,[0.5-5.71] ; \mathrm{p}=0.39)$ nor with $28^{\text {th }}$ day mortality (OR 2.17, [0.82-5.75]; $p=0.11$ ). Comparatively, the duration of mechanical ventilation and the ICU hospitalization period were less important in the hyperoxia group (Table 3 and Figure 2).

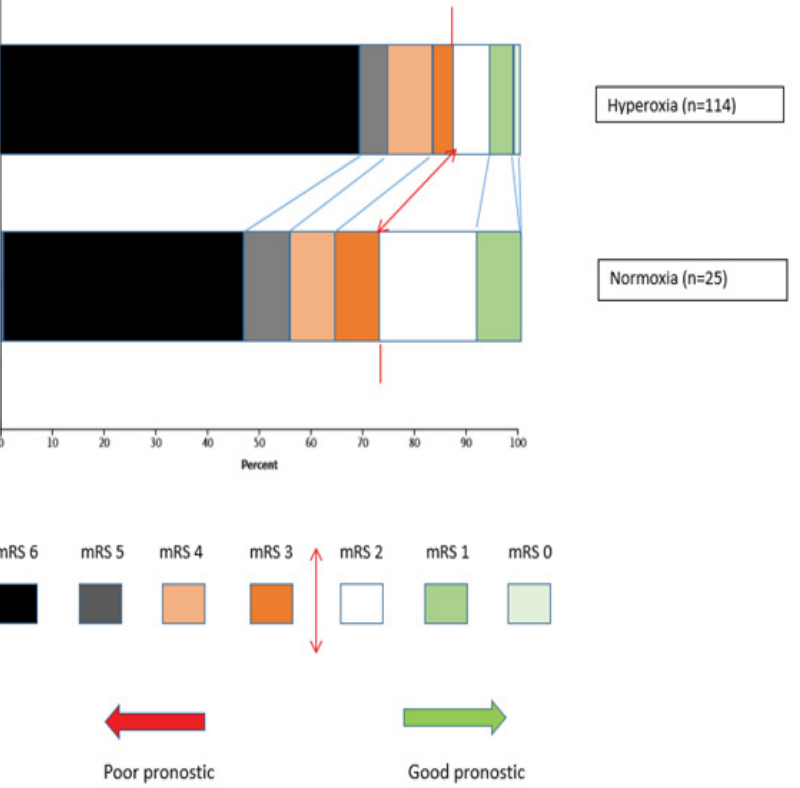

Figure 2 Modified Rankin scale (mRS) at 6 months. 
Table 1 General characteristics of the population.

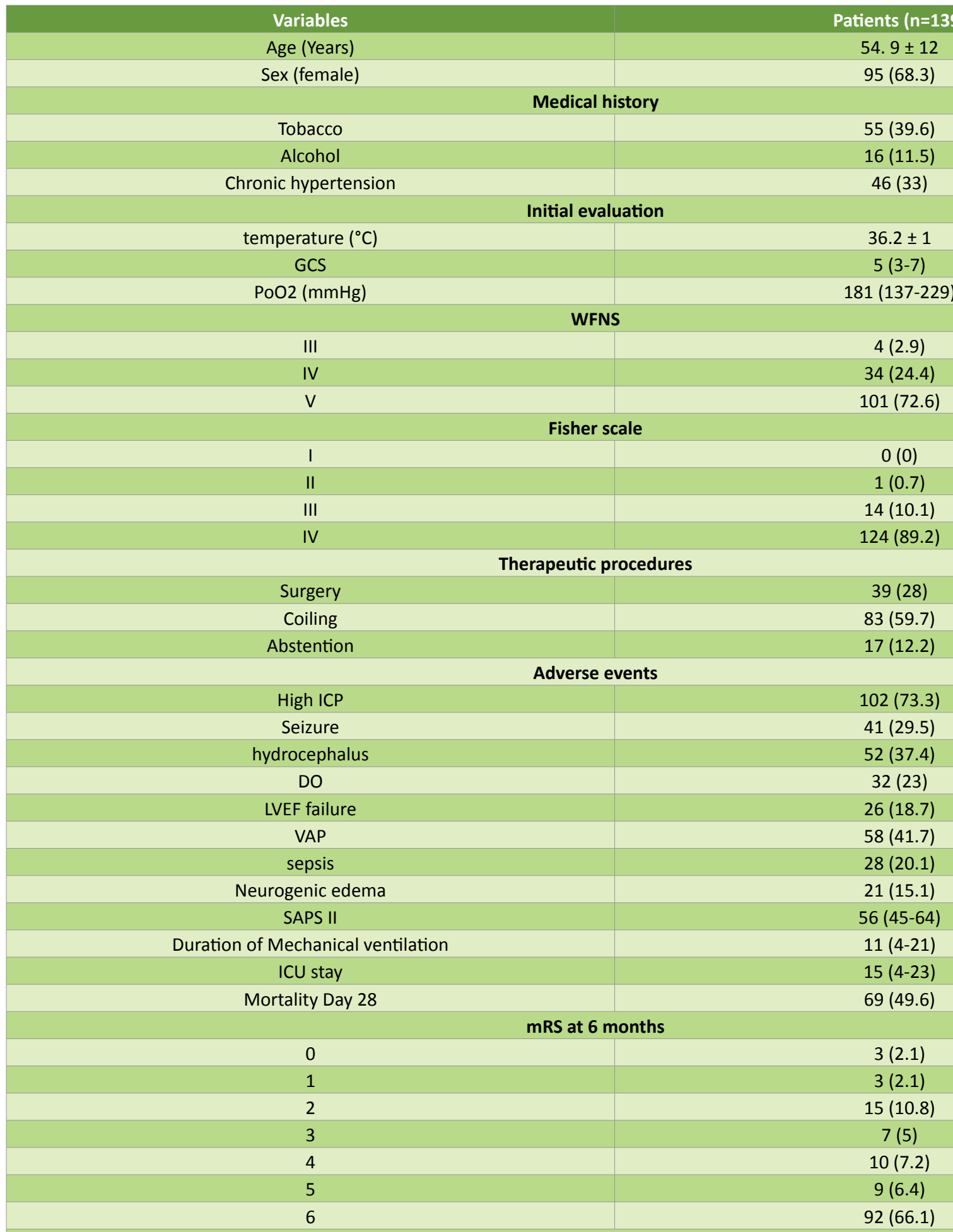

Data are expressed as mean \pm standard deviation. number (percentage). or median (interquartile range).

CGS: Glasgow Coma Scale; WFNS: World Federation of Neurosurgical Societies; ICP: Intracranial Pressure; DCI: Delayed Cerebral Ischemia; LVEF: Left Ventricular Ejection Failure; SAPS II: Simplified Acute Physiology Score; ICU: Intensive Care Unit; mRS: modified Rankin Scale

\section{Discussion}

Our study is the first to report a high trend association between Hyperoxia and 6-month poor neurological outcome in patients suffering from severe SAH admitted to the ICU. Our findings are in accordance with the results of the study published by Jeon et al. published in 2014, in this first and sole prospective study to ascertain this association, the authors noted the existence of a relationship, though non-statistical, between hyperoxia and a poor neurological outcome at 3 months $(p=0.087)$ In this study, only patients on mechanical ventilation were included. In addition, the study population was older ( 56.7 vs. 53.7 years of age $p=0.005$ ) and had more severe forms of meningeal bleeding 
Table 2 Sub-groups oxygenation.

\begin{tabular}{|c|c|c|c|c|c|}
\hline Variables & Normoxia $(n=25)$ & $\begin{array}{l}\text { Mild Hyperoxia } \\
\qquad(\mathrm{n}=60)\end{array}$ & $\begin{array}{l}\text { Moderate Hyperoxia } \\
(\mathrm{n}=42)\end{array}$ & $\begin{array}{l}\text { Severe Hyperoxia } \\
(n=12)\end{array}$ & $\mathbf{p}$ \\
\hline Age (Years) & $57.7 \pm 8.5$ & $55.5 \pm 11$ & $52.6 \pm 14.5$ & $54.1 \pm 12.8$ & 0.4 \\
\hline Sex (female) & $15(60)$ & $40(66.6)$ & $30(69.7)$ & $10(83)$ & 0.5 \\
\hline \multicolumn{6}{|c|}{ Medical history } \\
\hline Tobacco & $15(60)$ & $26(43.3)$ & $14(32.5)$ & $5(41.6)$ & 0.78 \\
\hline Alcohol & $8(32)$ & $6(10)$ & $7(16.2)$ & $0(0)$ & 0.48 \\
\hline Chronic hypertension & $10(40)$ & $20(33.3)$ & $14(32.5)$ & $4(33.3)$ & 0.99 \\
\hline \multicolumn{6}{|c|}{ Initial evaluation } \\
\hline $\mathrm{PaO} 2$ (mmHg) & $95(76-104)$ & $164(152-181)$ & $229(211-249)$ & $355(324-422)$ & $<0.0001$ \\
\hline Temperature $\left({ }^{\circ} \mathrm{C}\right)$ & $36.3 \pm 1$ & $36.3 \pm 1.1$ & $36.1 \pm 0.8$ & $35.6 \pm 1.1$ & 0.17 \\
\hline GCS & $7(4-8)$ & $4(3-7)$ & $5(3-8)$ & $3(3-8)$ & 0.25 \\
\hline \multicolumn{6}{|c|}{ WFNS } \\
\hline III & $0(0)$ & $2(3.3)$ & $1(2.4)$ & $3(8.3)$ & \multirow{3}{*}{0.0005} \\
\hline IV & $11(44)$ & $5(8.3)$ & $16(38.1)$ & $2(16.6)$ & \\
\hline V & $14(56)$ & $53(88.3)$ & $25(59.5)$ & $9(75)$ & \\
\hline \multicolumn{6}{|c|}{ Fisher test } \\
\hline 1 & $0(0)$ & $0(0)$ & $0(0)$ & $0(0)$ & \multirow{4}{*}{0.51} \\
\hline II & $1(4)$ & $0(0)$ & $0(0)$ & $0(0)$ & \\
\hline III & $1(4)$ & $8(13.3)$ & $4(9.6)$ & $1(8.3)$ & \\
\hline IV & $23(92)$ & $52(86.6)$ & $38(90.4)$ & $11(91.6)$ & \\
\hline \multicolumn{6}{|c|}{ Therapeutic procedures } \\
\hline Surgery & $5(20)$ & $19(31.6)$ & $9(21.5)$ & $6(50)$ & \\
\hline Coiling & $19(76)$ & $35(58.3)$ & $23(54.7)$ & $6(50)$ & 0.07 \\
\hline abstention & $1(4)$ & $6(10)$ & $10(23.8)$ & $0(0)$ & \\
\hline \multicolumn{6}{|c|}{ Adverse events } \\
\hline High ICP & $14(56)$ & $42(70)$ & $36(85.7)$ & $10(83)$ & 0.04 \\
\hline Seizure & $7(28)$ & $18(30)$ & $12(28.6)$ & $4(33.3)$ & 0.98 \\
\hline hydrocephalus & $10(40)$ & $22(36.6)$ & $15(35.7)$ & 5 (41.6) & 0.97 \\
\hline $\mathrm{DCl}$ & $4(25)$ & $14(23.3)$ & $13(30.9)$ & $1(8.3)$ & 0.3 \\
\hline LVEF failure & $5(20)$ & $12(20)$ & $8(19)$ & $1(8.3)$ & 0.89 \\
\hline VAP & $11(44)$ & $26(43.3)$ & $17(40.4)$ & $4(33.3)$ & 0.92 \\
\hline Neurogenic edema & $5(20)$ & $10(16.6)$ & 5 (11.9) & $1(8.3)$ & 0.76 \\
\hline sepsis & $5(20)$ & $13(21.6)$ & $9(21.4)$ & $1(8.3)$ & 0.76 \\
\hline SAPS II & $55(43-66)$ & $56(44-63)$ & $54(44-62)$ & $55(48-72)$ & 0.8 \\
\hline Duration of Mechanical ventilation & $12(9-19)$ & $14(4-23)$ & $11(3-19)$ & $5(2-18)$ & 0.48 \\
\hline ICU stay & $16(10-22)$ & $15(4-22)$ & $12(3-21)$ & $6(2-23)$ & 0.44 \\
\hline Mortality Day 28 & $8(32)$ & $33(55)$ & $22(51)$ & $7(58.3)$ & 0.24 \\
\hline \multicolumn{6}{|c|}{ Prognostic at 6 months } \\
\hline Good prognostic (mRS<3) & $7(28)$ & $7(11.6)$ & $4(9.75)$ & $3(25)$ & \multirow{2}{*}{0.05} \\
\hline Poor prognostic (mRS $\geq 3$ ) & $18(72)$ & $53(88.4)$ & $38(90.25)$ & $9(25)$ & \\
\hline
\end{tabular}

Data are expressed as mean \pm standard deviation. Number (percentage) or median (Interquartile range).

CGS: Glasgow Coma Scale; WFNS: World Federation of Neurosurgical Societies; ICP: Intracranial Pressure; DCI: Delayed Cerebral Ischemia; LVEF: Left Ventricular Ejection Failure; SAPS II: Simplified Acute Physiology Score; ICU: Intensive Care Unit; mRS: modified Rankin Scale.

Table 3 Univariate and multivariate analysis.

\begin{tabular}{|c|c|c|c|c|c|c|}
\hline \multirow{2}{*}{ Variables } & \multicolumn{3}{c}{ Univariate analysis } & \multicolumn{2}{c|}{ Multivariate analysis } \\
\hline & Normoxia $(\mathbf{n = 2 5})$ & Hyperoxia (n=114) & OR (95 IC) & P-value & OR (95\% Cl) & P-value \\
\hline Poor neurologic outcome (mRS $\geq 3)$ & $18(72)$ & $100(87.7)$ & $2.8(1.02-7.71)$ & 0.12 & $2.84(0.99-8.19)$ & 0.052 \\
\hline Delayed Cerebral Ischemia & $4(16)$ & $28(24.6)$ & $1.7(0.55-5.35)$ & 0.43 & $1.68(0.5-5.71)$ & 0.39 \\
\hline Mortality day-28 & $8(32)$ & $62(54.3)$ & $2.5(0.93-7.31)$ & 0.05 & $2.17(0.82-5.75)$ & 0.11 \\
\hline Duration of mechanical ventilation & $12(9-19)$ & $11(3-21)$ & -- & 0.47 & -- & -- \\
\hline ICU-Stay & $16(10-22)$ & $14(3-23)$ & -- & 0.38 & -- \\
\hline
\end{tabular}

compared to patients excluded from the study (Glasgow, Hunt and Hess score, modified Fisher score $p<0.001$ ) [9]. On the other hand, a more recently published retrospective cohort study did not find a correlation between hyperoxia and the emergence of 
a poor neurological fallout ( $p=0.06)$. In contrast, in this study, the subgroup of patients with H\&K I-III SAH exposed to hyperoxia had an unfavorable neurological prognosis $(p=0.02)$ [10].

In our population the hyperoxia was very early, because patients were often admitted to the unit already under mechanical ventilation with pre-hospital orotracheal intubation, with frequent transfer under high levels of $\mathrm{FiO}_{2}$. More generally, hyperoxia has been associated with a higher mortality in other diseases. A study examining the effect of hyperoxia on brain injury found an increase in mortality [2]. Another cohort study reviewing patients resuscitated from a cardiac arrest, found an association between hyperoxia and an increase in hospital mortality [1]. Elsewhere, other studies have claimed a protective cerebral effect of hyperoxia in patients presenting with a stroke or brain injury. Hyperoxia would improve brain metabolism by lowering lactate levels and therefore enhancing cerebral neuroenergetics [14].

The harmful mechanisms of hyperoxia are controversial and are currently the subject of much debate. However, studies have shown that hyperoxia increases the amount of oxygen dissolved in the blood causing the overproduction of oxygen derivatives including free radicals. These free radicals hold a free electron on their surface and are therefore extremely reactive with their environment [15].

Physiologically, mitochondria produce free radicals, but they are quickly destroyed. Their production leads to lipid peroxidation, protein oxidation and DNA damage. Peroxidation leads to enzymatic inactivation, oxidation of thiol proteins and inhibition of the mitochondrial respiratory chain [16]. Protein oxidation results in the formation of protein aggregate by resistance to proteolysis [17]. Finally, free radicals hold DNA toxicity, leading to apoptosis and carcinogenesis [18]. Other studies have suggested that hyperoxia may lead to vasoconstriction and decreased cerebral blood flow. Ultimately, this oxidative stress leads to cardiac, pulmonary and neurological toxicity [19]. Hyperoxia is also harmful at the pulmonary level with the appearance of Hyperoxic Acute Lung Injury (HALI) [20], secondary to mechanical ventilation at a high level of $\mathrm{FiO}_{2}$ that can create real respiratory distress and thus worsen the prognosis of SAH patient.

The results of our study may be explained by the definition choice of hyperoxia. Indeed, the 2 previously mentioned studies had a divergent cutoff point for defining hyperoxia and had thus contrary findings. We decided to classify hyperoxia into three subgroups, as done in the most recent study by Yokoyama et al. regardless of the lack of a statistical link in their findings. Our study did not substantiate a significant difference regarding the occurrence of vasospasms in the subgroup analysis $(p=0.3)$.

\section{References}

1 Kilgannon JH, Jones AE, Shapiro NI, Angelos MG, Milcarek B, et al. (2010) Association between arterial hyperoxia following resuscitation from cardiac arrest and in-hospital mortality. JAMA 303: 2165-2171.

2 Brenner M, Stein D, Hu P, Kufera J, Wooford M, et al. (2012) Association between early hyperoxia and worse outcomes after traumatic brain injury. Arch Surg 147: 1042.
Likewise, the study by Yokoyama et al. found no association between a hyperoxic state and the development of ischemic events [10]. However, it is interesting to note that their $\mathrm{PaO}_{2}$ threshold for defining hyperoxia was $<120 \mathrm{mmHg}$. In contrast, the moderate hyperoxic group in our study was defined by a $\mathrm{PaO}_{2}$ level $>180 \mathrm{mmHg}$. In comparison, the prospective study by Jeon et al. fixed the $\mathrm{PaO}_{2}$ threshold at $>173 \mathrm{mmHg}$ and found that hyperoxia caused a greater number of vasospasms among their study patients $(p=0.001)$ [9].

\section{Limitations}

Our study has several limitations. We conducted a monocentric, retrospective study, and only a small number of patients were included, thus hampering the interpretation of our results. It is conceivable that, given the statistical trend found in our analysis, a more substantial study population could yield significant statistical results, and render our hypothesis factual. Indeed, patients presenting with a high grade SAH (WFNS $\geq 3$ ) were only included, and the absence of confounding factors, between a worse WFNS score and the emergence of a poor prognosis, under these circumstances, cannot be verified with certainty. In addition, $\mathrm{PaO}_{2}$ levels were only measured upon ICU admission. This single value does not reflect prolonged exposure to hyperoxia. However, one study showed that an early exposure to hyperoxia in the first few hours of medical care was more harmful than delayed exposure [20]. Furthermore, we were not able to analyze hyperoxia by other techniques such as interstitial brain tissue oxygenation by $\mathrm{PbtO}_{2}$ probe or by Near Infrared Spectroscopy (NIRS), instead of $\mathrm{PaO}_{2}$ levels [21-23].

\section{Conclusion}

In conclusion, our study did find a significant association between the early exposure to hyperoxia and an unfavorable neurological evolution at 6 months; in patients with a high grade aneurysmal $\mathrm{SAH}$. Our findings suggest the existence of a statistical trend between both variables, mainly due to a modest patient sample size, thus lowering its statistical power. At present, there is no standard definition of hyperoxia. A large-scale, multicentric, prospective study would serve to determine the underlying effects of hyperoxia on the long-term neurological prognosis, and to pinpoint $\mathrm{a} \mathrm{PaO}_{2}$ threshold leading to such effects, and not to exceed.

\section{Conflicts of Interest}

None.

\section{Sources of Funding}

None.

3 Janz DR, Hollenbeck RD, Pollock JS, McPherson JA, Rice TW (2012) Hyperoxia is associated with increased mortality in patients treated with mild therapeutic hypothermia after sudden cardiac arrest. Crit Care Med 40: 3135-3139.

4 Stub D, Smith K, Bernard S, Nehme Z, Stephenson M, et al. (2015) Air versus oxygen in ST-segment-elevation myocardial infarction. Circulation 131: 2143-2150. 
5 European Registers of Stroke (EROS) Investigators, Heuschmann PU, Di Carlo A, Bejot Y, Rastenyte D, et al. (2009) Incidence of stroke in Europe at the beginning of the 21st century. Stroke 40: 1557-1563.

6 Connolly ES, Rabinstein AA, Carhuapoma JR, Derdeyn CP, Dion J, et al. (2012) Guidelines for the management of aneurysmal subarachnoid hemorrhage: a guideline for healthcare professionals from the American Heart Association/American Stroke Association. Stroke 43: 1711-1737.

7 Diringer MN, Bleck TP, Claude HJ, Menon D, Shutter L, et al. (2011) Critical care management of patients following aneurysmal subarachnoid hemorrhage: Recommendations from the Neurocritical Care Society's Multidisciplinary Consensus Conference. Neurocrit Care 15: 211-240.

8 Frontera JA, Fernandez A, Schmidt JM, Claassen J, Wartenberg KE, et al. (2009) Defining vasospasm after subarachnoid hemorrhage: What is the most clinically relevant definition? Stroke 40: 1963-1968.

9 Jeon SB, Choi HA, Badjatia N, Schmidt JM, Lantigua H, et al. (2014) Hyperoxia may be related to delayed cerebral ischemia and poor outcome after subarachnoid haemorrhage. J Neurol Neurosurg Psychiatry 85: 1301-1307.

10 Yokoyama S, Hifumi T, Kawakita K, Tamiya T, Minamino T, et al. (2018) Early hyperoxia in the intensive care unit is significantly associated with unfavorable neurological outcomes in patients with mild-to-moderate aneurysmal subarachnoid hemorrhage. Shock 51: 593-598.

11 Leone M, Bouadma L, Bouhemad B, Brissaud O, Dauger S, et al. (2018) Brief summary of French guidelines for the prevention, diagnosis and treatment of hospital-acquired pneumonia in ICU. Ann Intensive Care 8: 104

12 Davison DL, Terek M, Chawla LS (2012) Neurogenic pulmonary edema. Crit Care 16: 212.

13 Rhodes A, Evans LE, Alhazzani W, Levy MM, Antonelli M, et al. (2017)
Surviving sepsis campaign: International guidelines for management of sepsis and septic shock 2016. Intensive Care Med 43: 304-377.

14 Tolias CM, Reinert M, Seiler R, Gilman C, Scharf A, et al. (2004) Normobaric hyperoxia induced improvement in cerebral metabolism and reduction in intracranial pressure in patients with severe head injury: A prospective historical cohort-matched study. J Neurosurg 101: 435-444.

15 Liaudet L (2007) Oxidative biology and clinical implications of peroxynitrite. Rev Med Suisse 3: 2840-2843.

16 Niki E (2009) Lipid peroxidation: Physiological levels and dual biological effects. Free Radic Biol Med 47: 469-484.

17 Berlett BS, Stadtman ER (197) Protein oxidation in aging, disease, and oxidative stress. J Biol Chem 272: 20313-20316.

18 Nathan C, Cunningham-Bussel A (2013) Beyond oxidative stress: An immunologist's guide to reactive oxygen species. Nat Rev Immunol 13: 349-361.

19 Llitjos JF, Cariou A (2018) Effets de l'hyperoxie sur le pronostic après un arrêt cardiaque. Médecine Intensive Réanimation 27: 133-142.

20 Kallet RH, Matthay MA (2013) Hyperoxic acute lung injury. Respir Care 58: 123-141.

21 Rosenthal RE, Silbergleit R, Hof PR, Haywood Y, Fiskum G (2003) Hyperbaric oxygen reduces neuronal death and improves neurological outcome after canine cardiac arrest. Stroke 34: 1311-1316.

22 Keller E, Froehlich J, Baumann D, Böcklin C, Sikorski C, et al. (2015) Detection of delayed cerebral ischemia (DCI) in subarachnoid haemorrhage applying near-infrared spectroscopy: Elimination of the extracerebral signal by transcutaneous and intraparenchymatous measurements in parallel. Acta Neurochir Suppl 120: 243-247.

23 Stambolija V, Miklić BM, Lozić M, Nemir J, Ščap M (2018) PbtO2 monitoring in normobaric hyperoxia targeted therapy in acute subarachnoidal hemorrhage. Surg Neurol Int 9: 46. 